\title{
Mesothelioma developing in carriers of inherited genetic mutations
}

\author{
Yoshie Yoshikawa $^{1}$, Mitsuru Emi ${ }^{1,2}$, Takashi Nakano ${ }^{3}$, Giovanni Gaudino ${ }^{2}$ \\ ${ }^{1}$ Department of Genetic, Hyogo College of Medicine, Nishinomiya, Hyogo, Japan; ${ }^{2}$ University of Hawai'i Cancer Center, Honolulu, HI, USA; \\ ${ }^{3}$ Center for Respiratory Medicine, Otemae Hospital, Chuo-ku, Osaka, Japan \\ Contributions: (I) Conception and design: Y Yoshikawa, M Emi, G Gaudino; (II) Administrative support: T Nakano; (III) Provision of study materials \\ or patients: T Nakano; (IV) Collection and assembly of data: Y Yoshikawa; (V) Data analysis and interpretation: Y Yoshikawa, M Emi, G Gaudino; (VI) \\ Manuscript writing: All authors; (VII) Final approval of manuscript: All authors. \\ Correspondence to: Dr. Yoshie Yoshikawa. Hyogo College of Medicine, Nishinomiya, Hyogo J663-8501, Japan. Email: yoshiey@hyo-med.ac.jp.
}

\begin{abstract}
Malignant mesothelioma is associated with the exposure to asbestos fibers. Recent discovery of the $B A P 1$ cancer syndrome, a Mendelian disorder with high-penetrance autosomal dominant inheritance fostered the genotyping for nucleotide-level or larger structural alteration of germline DNA. Inherited heterozygous mutations of the $B A P 1$ gene increase the susceptibility to carcinogenic fibers, leading to a concept of gene $\mathrm{x}$ environment interaction $(\mathrm{GxE})$ as a pathogenetic mechanism of mesothelioma. Several studies on cohorts of unselected patients with mesothelioma or on familial/early-onset cohorts of mesothelioma cases converged on BAP1 as the more frequent germline mutated gene, followed by other genes involved in DNA repair and homologous recombination. Evidence has been emerging that patients with mesothelioma carrying germline mutations of BAP1 and of other genes, such as those involved in DNA repair and tumor suppressor genes, have better prognosis and higher chemosensitivity when compared with patients with germline wildtype Bap1. We report here a germline genomic analysis targeted 22 genes in a cohort of 101 Japanese patients irrespective of asbestos exposure, age at diagnosis, or personal or family history of cancer. By comparing the results with the Human Genetic Variation Database (HGVD) and the Genome Aggregation Database (gnomAD) we selected rare germline variants with a Combined Annotation Dependent Depletion (CADD) >20. We show here that 31 of 101 subjects were carrying 25 rare variants in 14 genes, neither reported in the HGVD nor in the gnomAD database for 14/25 variants. Besides pathogenic variants of $B A P 1$, rare missense variants were found in genes encoding lysine-specific histone methyltransferase SETD2 and SETDB1 and genes encoding subunits of the mSWI/SNF chromatin remodeling complex. The complete scenario of the genetic background consisting of pathogenic germline variants required for the predisposition and GxE for pathogenesis of mesothelioma appears complex, and further large-scale studies are warranted.
\end{abstract}

Keywords: DNA repair genes; inherited mutations; mesothelioma; pathogenic variants

Submitted Aug 27, 2019. Accepted for publication Nov 13, 2019.

doi: $10.21037 /$ tlcr.2019.11.15

View this article at: http://dx.doi.org/10.21037/tlcr.2019.11.15

\section{Introduction}

Malignant mesothelioma has long been known as cancer closely linked to occupational exposure to asbestos fibers. A significantly higher incidence of mesothelioma compared with the general population was found among workers handling asbestos, like miners (1), workers in shipyards (2), and even workers' relatives or residents in areas near the asbestos factories (3). Asbestos is a general term used in the mineral commodity field to identify 5 amphibole and 1 serpentine silicate fibers that were extensively used until the end of the last century when asbestos was banned or strictly 
regulated in the Western world and Japan. In contrast, asbestos is still in use in several developing countries, where the incidence of mesothelioma is expected to increase in the next years (4). However, an additional risk originates from the exposure to the approximately further 400 silicates that naturally occur worldwide, which may have even more powerful activity than asbestos in inducing mesothelioma, as it was described for erionite $(5,6)$.

Human mesothelial cells lining the pleural, pericardial and peritoneal cavities are particularly susceptible to cytotoxicity induced by asbestos fibers and it was demonstrated that after exposure to the amphibole crocidolite fibers the major component of cell death is necrosis (7). Similar results were obtained by exposing cells to erionite (6) fibers or to serpentine chrysotile fibers, which have reduced biopersistence accompanied by lower pathogenicity (8). However, asbestos cytotoxicity consists of a regulated form of necrosis that results in the passive release of the damage-associated molecular protein (DAMP) high mobility group box 1 (HMGB1), recruiting macrophages that propagate chronic inflammation $(7,9)$. Due to this pro-inflammatory environment, the mesothelial cells that have survived the asbestos exposure and accumulated genetic alterations in the genomes may give rise to mesothelioma over a period of many years (10).

\section{Mesothelioma is linked to gene $x$ environment interaction (GXE)}

A large body of evidence has shown among the workers with a long history of exposure to asbestos, only $~ 5 \%$ developed mesothelioma (4). Moreover, the discovery that the susceptibility to mesothelioma was transmitted in Mendelian autosomal dominant inheritance upon exposure to erionite $(11,12)$ indicated mesothelioma as a model of GxE. According to this model, individuals carrying germline alterations to one or more genes may be more susceptible to fiber-induced carcinogenesis and to the development of this type of cancer.

Array-comparative genomic hybridization (aCGH) and linkage analysis in samples from two unrelated families in the US ( $L$ from Louisiana and $W$ from Wisconsin), with no reported exposure to asbestos but an unusually high incidence of mesothelioma, pointed at chromosome $3 \mathrm{p} 21$, where sequence analysis identified germline $B A P 1$ mutations associated with autosomal dominant transmission of mesothelioma and uveal melanoma (13). By using a similar approach, a synchronous paper from a different group identified germline mutations of $B A P 1$ as responsible for dominant inheritance of melanocytic tumors (14). Further support to the role of BAP1 in predisposing to mesothelioma came from experiments performed with $B a p 1^{+/}$heterozygous mice exposed to very low doses of asbestos fibers. These animals developed mesothelioma at a comparable rate of wild type Bap1 mice when they were exposed to asbestos at ten-time higher doses (15).

Additional evidence from different groups contributed to the association of germline BAP1 mutations with the increased risk of atypical Spitz tumors and of uveal melanoma, pleural mesothelioma (16-18), peritoneal mesothelioma (19-21), clear cell renal cell carcinoma $(22,23)$, cutaneous melanoma, ocular melanoma (24), basal cell carcinoma $(25,26)$, meningioma $(27)$, and cholangiocarcinoma (28). This substantial body of evidence has led to developing the concept of the BAP1 cancer syndrome, a Mendelian disorder, with high-penetrance autosomal dominant inheritance (29). Interestingly, BAP1 mutations were identified in a large cohort of FrenchCanadian families at high-risk for breast cancer with no mutations in BRCA1 and BRCA2 genes (30), suggesting that some portion of breast cancers may also belong to the spectrum of tumors associated with the BAP1 cancer syndrome (31). Recent data from a large worldwide analysis of BAP1 germline variants and of the clinical phenotypes associated with the BAP1 cancer syndrome, confirmed that in addition to the four core syndrome tumors (uveal melanoma, malignant mesothelioma, cutaneous melanoma, and clear cell renal carcinoma), also non melanoma skin tumors (mostly basal cell carcinoma), meningioma, and cholangiocarcinoma were present in some of the families analyzed in the study (32).

The discovery of the BAP1 syndrome underscores the importance of genotyping cancer patients for nucleotidelevel mutations and gross structural alterations of germline DNA, to determine the presence of germline mutations in yet un-linked additional genes for the identification of inherited predisposition to GxE-dependent cancers, like mesothelioma $(20,33,34)$.

The most recent literature on the germline genomic analysis in mesothelioma was reviewed here. Moreover, novel germline alterations in a population of Japanese patients with mesothelioma were also reported.

\section{Inherited predisposition to cancer}

The effect of GxE interaction on cancer development is 
driven not only by the accumulation of spontaneous somatic mutations due to the exposure to carcinogenic substances (as in the case of asbestos and other carcinogenic fibers), but also by the presence of inherited mutations occurring in genes that are critical for the genome homeostasis, like those regulating DNA repair and/or homologous recombination (HR). When these genes are mutated the susceptibility to carcinogens would increases, leading to a higher frequency of DNA damage and gene alterations. As a consequence, more cells would accumulate mutations in these individuals, who become predisposed to develop certain types of cancer, and the genes with these pathogenic, germline, inherited mutations are to be associated with cancer syndromes (4). The diversity of tissue specificity and prevalence of the related tumors shall be also determined by the function that is disrupted by the gene mutation, and by the specific context of the $\mathrm{GxE}$ interaction involved. Moreover, given the multifactorial nature of cancer, when genetic background and carcinogen exposure are evaluated, the difference between the causative role of exposure and its association with carcinogenesis must be carefully taken into account (35).

Powerful tools for nucleic acid sequence analysis, like next-generation sequencing (NGS), whole-genome sequencing (WGS), whole-exome sequencing (WES), and gross structural analysis methods as well, such as multiplex ligation-dependent probe amplification (MLPA) and array $\mathrm{CGH}$, were used to conduct extensive genetic analyses of a large number of genes in germline DNA (as well as in somatic DNA from cancer samples). This approach allowed the identification of germline pathogenic variants in several genes and to associate these variants to a higher risk of several types of cancer. Examples of genes responsible for cancer syndromes are BAP1 (29), TP53 (36), and BLM (37), among others. Further analysis will expand the spectrum of inherited pathogenic variants of cancer predisposition genes, with the aim of promoting massive programs of genetic screening of the population.

\section{Germline mutation analysis in mesothelioma: what it is known}

The discovery of $B A P 1$ as a predisposition gene to mesothelioma and other cancers (29) paved the way for germline analysis in patients with mesothelioma and individuals at high risk of mesothelioma (e.g., because of environmental exposure to carcinogenic fibers). The number of these studies, aimed at investigating the prevalence of germline variants in cancer susceptibility genes, have exponentially increased in the recent past years.

Two studies from the same group evaluated the prevalence of pathogenic germline variants in patients with pleural mesothelioma in association with cumulative asbestos exposure that was assessed according to the data from an Italian mesothelioma registry, using a standardized face-to-face questionnaire. The calculated estimate of long-life exposure was expressed as fibers/milliliter-years (f/mL-years, a measure of cumulative exposures in terms of the concentration of fibers in the air over time). Eightynine patients with mesothelioma were recruited and among these patients, 34 had a documented history of familial mesothelioma or of other tumors included in the BAP1 cancer syndrome. Ninety-four cancer-predisposing genes were analyzed as target genes (albeit 6 patients with familiarity for cancer were screened for only 6 genes). Truncating (nonsense and frameshift) germline variants were selected as pathogenic truncating variants (PTVs). Four patients with mesothelioma had BAP1 germline variants, including non-PTV at the canonical splicing site and 10 patients carried germline PTVs in other tumor suppressor genes: $C D K N 2 A$ and DNA repair genes. A significant difference $(\mathrm{P}=0.00002)$ was found between the group of patients with familial mesothelioma and pathogenic germline variants in tumor suppressor genes who had lower asbestos exposure $(n=14$, mean $=-1.299$ $\mathrm{f} / \mathrm{mL}$-years, SD =2.17), compared with the group of patients with no germline variants in the 94 cancerpredisposing genes $(n=67$, mean $=1.093 \mathrm{f} / \mathrm{mL}$-years, SD =1.71). Moreover, the cumulative asbestos exposure was significantly lower in patients with familial mesothelioma, including patients carrying mutations and patients with no known germline variants $(n=34$, mean $=0.000015$, SD =2.02), when compared to patients who had neither familial mesothelioma, nor germline variants in the 94 cancer-predisposing genes $(\mathrm{n}=55$, mean $=1.092, \mathrm{SD}=1.82)$ $(\mathrm{P}=0.01)(38,39)$.

A different study performed targeted NGS (tNGS) of 85 cancer susceptibility genes in germline DNA from 198 patients with pleural, peritoneal, and tunica vaginalis mesothelioma (20). The frequency of the variants within the population was established by using the publicly available data of the Exome Aggregation Consortium (ExAC) and the results were interpreted according to the American College of Medical Genetics and Genomics/ Association for Molecular Pathology (ACMG/AMP) guidelines (40). In 23 of 198 patients with mesothelioma 
(12\%), germline mutations were present in 13 cancer susceptibility genes, HR DNA repair genes in particular. Family history of cancer and asbestos exposure were also assessed by a standardized questionnaire. The extent of selfreported asbestos exposure was classified in 4 levels: definite, probable, possible, and no known. The results of multivariate analysis showed that minimal-to-no asbestos exposure was the most significant predictor of the presence of a germline cancer susceptibility mutation. Moreover, having a younger age or a second cancer at diagnosis were further significant predictors of the occurrence of germline cancer susceptibility mutations (20).

To identify mesothelioma associated with germline mutations of $B A P 1$ or other genes, germline mutations in $B A P 1$ were screened by Sanger sequencing, and in 55 additional genes linked to cancer by tNGS in a population of 79 familial pleural or peritoneal mesothelioma cases, consisting of unrelated probands $(\mathrm{n}=52)$ and their firstand second-degree relatives $(n=27)$ (33). The patients of this population were selected according to four predefined criteria suggesting heritability of $B A P^{+/}$status: (I) first- or second-degree relatives with mesothelioma; (II) proband or at least one first- or second-degree relative diagnosed with malignancies that frequently occur in $B A P 1^{+/}$carriers (uveal melanoma, cutaneous melanoma, clear-cell renal cell carcinoma); (III) history of multiple cancers (any cancer) in the majority of first- and second-degree relatives; (IV) early onset of mesothelioma (age $<50$ years). Familial mesothelioma patients who carried $B A P 1^{+/-}$represented the largest subpopulation of the enrolled patients (43/79). tNGS analysis was performed in 45 patients with mesothelioma (34 wildtype $B A P 1$ and $11 B A P 1^{+/}$) for 56 cancer-linked genes (including BAP1). Only mutations with an allele frequency less than 0.005 in the ExAC database and with the stringent cut-off of a Combined Annotation Dependent Depletion (CADD; http://cadd.gs.washington. edu/) score greater than 20 were considered. The results of the tNGS analysis showed that 12 of 34 patients with mesothelioma having wildtype BAP1 (35\%) carried one germline mutation in 11 genes and 5 of $11 \mathrm{BAP}^{+/}$patients with mesothelioma ( $45 \%$ ) carried one additional germline mutation in 5 different genes. The deleterious germline variants identified in the patients selected by our four familial/early-onset criteria involved cancer susceptibility genes, as ARID1A, ARID2, BAP1, CREBBP, KDR, MLH1, NCOR1, RAD50, RBM6, SETD2, SMARCA2, SMARCA4, SMARCE1, SMO, TP53. The available data on survival of the patients of this study $(\mathrm{n}=77)$ were compared with those of the mesothelioma dataset from the Surveillance, Epidemiology, and End Results (SEER) Program (https:// seer.cancer.gov). Interestingly, the cohort of patients selected by using the four criteria above had a remarkably significant improvement of survival and earlier age at diagnosis, compared with the SEER cohort. The median survival and age at diagnosis were 5 and 54 years, respectively, among the patients with $B A P 1^{+/}$, and 9 and 45 years among ones with wildtype $B A P 1$, whereas 8 months median survival and 72 years at diagnosis were found in the SEER cohort (33). These results suggest that the chosen selection criteria may help to identify the patients and their relatives who are susceptible to develop additional cancer and should be offered genetic counseling and a cancer prevention approach.

A total of 385 patients with pleural, peritoneal, pericardial, or tunica vaginalis mesothelioma were enrolled from the two large centers of the National Cancer Institute (NCI) and the University of Chicago (UC), to evaluate the link between germline mutations in tumor suppressor or DNA repair genes and the response to platinumbased chemotherapy in terms of survival. The multi-gene panel BROCA v10, including 73 target genes linked to DNA repair and/or to inherited predisposition to develop solid tumors was used. Frameshifts, nonsense, complete gene deletions, splice mutations, or missense mutations were deemed damaging mutations, corresponding to experimentally established losses of transcripts or function. In the NCI cohort, 28 of the 239 unrelated patients (12\%) carried a damaging mutation in one of the targeted genes: 16 mutations involved $B A P 1$ and 12 were disseminated among other genes, namely CHEK2, PALB2, BRCA2, MLH1, POT1, TP53, and MRE11A. The association of patient genotype with overall survival (OS) was evaluated for all 385 patients from the combined cohorts who were treated with platin chemotherapy. In patients carrying a mutation in $B A P 1$, or with a mutation in any of the targeted genes, OS was significantly improved, as compared with patients with no germline mutations (median OS $8.0 \mathrm{vs}$. 2.9 years, $\mathrm{P}=0.004$ in $B A P 1$ mutated patients; median OS 8.0 vs. 2.9 years, $\mathrm{P}=0.0006$ in patients carrying target gene mutations). Interestingly, this difference related to the genotype was highly significant in patients with pleural mesothelioma, but not in patients with peritoneal mesothelioma (41).

A tNGS approach spanning whole exon regions of each gene was used to investigate germline variants in 636 patients with different advanced solid cancers, including 
Table 1 Summary of the most recent studies on germline genetic alterations in mesothelioma

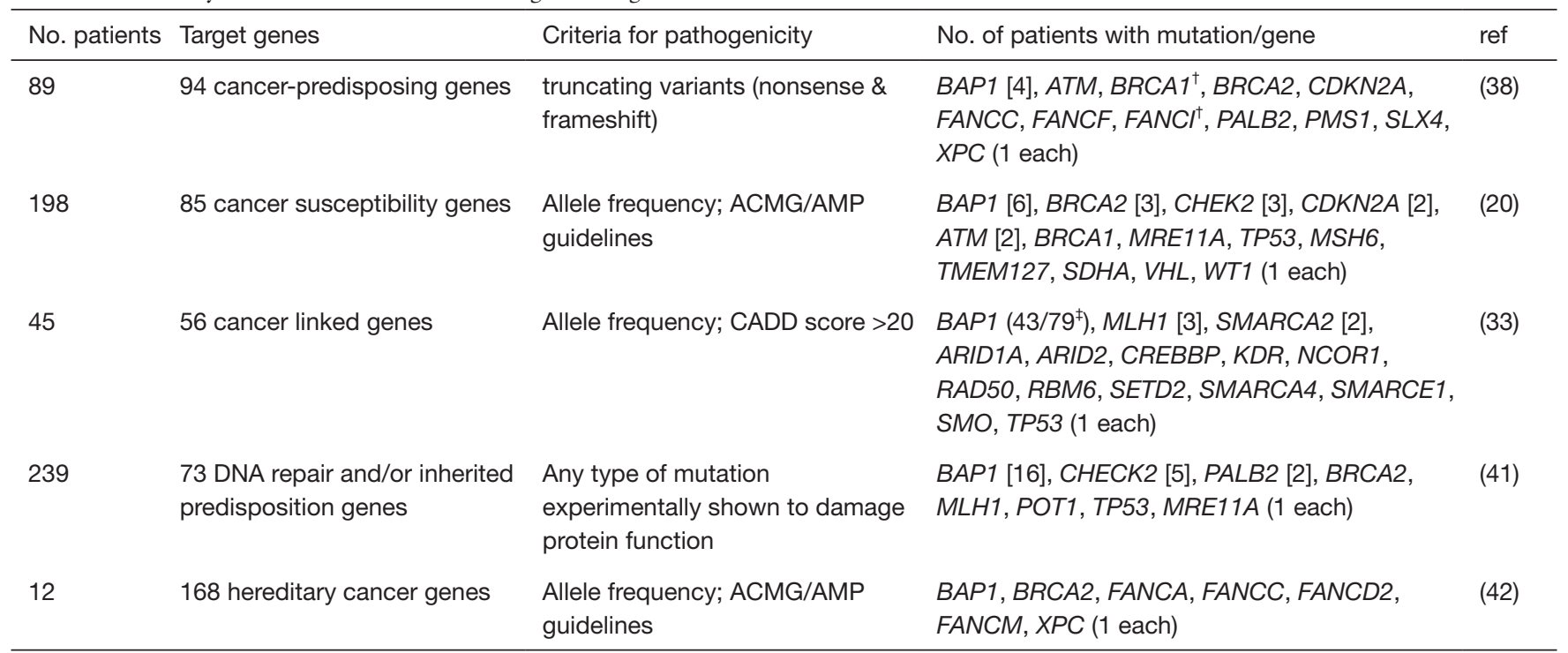

${ }^{\dagger}$, detected in the same patient; ${ }^{\ddagger}$, detected in 16 probands with $\mathrm{BAP}^{+/-}$and 27 relatives.

12 mesotheliomas. A large panel of 168 genes associated with hereditary cancer was analyzed and germline variants were filtered and classified using the commercially available Ingenuity Variant Analysis (IVA) software. The variants were then manually classified as pathogenic or likely pathogenic in this large patient cohort, according to the ACMG/AMP recommendations (40) by using the information about the allele frequency, in-silico functional analysis tools, and literature search. Patients with mesothelioma represented a small fraction of the large cohort examined (12/636 cases), however, this subpopulation displayed the highest frequency of pathogenic variants $(7 / 12,58 \%)$. Other solid tumors, like ovarian cancer $(7 / 23,30 \%)$, cervical cancer $(5 / 18,28 \%)$, urothelial cancer $(5 / 20,25 \%)$, and cancer of unknown primary origin $(4 / 16,25 \%)$, followed with lower frequencies. The germline variants were mostly present in genes regulating DNA repair. The variants in HR repair genes were mostly represented in mesothelioma and in particular mutations affecting the genes of Fanconi anemia pathway (BRCA2 or FANCD1, FANCA, FANCC, FANCD2, and FANCM) (42).

A summary of these studies is shown in Table 1 and all of them indicate that at least $10-12 \%$ of mesotheliomas are dependent upon the genetic background of the patients, with germline mutations in BAP1 or in HR genes, conferring to the patients better prognosis and chemosensitivity than in germline wildtype patients. However, more research will be needed to identify other genes, beyond the HR-related ones, which may be involved in the predisposition to developing mesothelioma. Furthermore, the criteria to evaluate the clinical significance of the germline variants identified should be improved, as many variants are still under pending judgment as variants of uncertain significance (VUS).

\section{Chromothripsis in mesothelioma and germline alterations}

The catastrophic genetic event known as chromothripsis consists of the fragmentation of a segregated single chromosome that is then rearranged leading to incorrect reassemble or loss of certain DNA sequence. Therefore, a single chromothripsis event may cause a high number of alterations in the genome after a short number of cell replications, leading to oncogenic activations or to loss of tumor suppressor functions, eventually favoring tumorigenesis (43).

Interestingly, non-contiguous biallelic genome alterations with the characteristic pattern of chromothripsis have been observed in mesothelioma (44), later confirmed by other groups (45), also with the potential consequence of neoantigen expression and tumor immunogenicity (46).

These studies were conducted in tumor samples and focusing on somatic mutations. However, a possible link between germline DNA alterations and chromothripsis in mesothelioma can be postulated. First, the accumulation 
Table 2 Demographic, clinical, and pathology data for the Japanese population under study $(\mathrm{n}=101)$

\begin{tabular}{|c|c|}
\hline Patient characteristics & No. of patients (\%) \\
\hline \multicolumn{2}{|l|}{ Gender } \\
\hline Male & $76(75.2)$ \\
\hline Female & $25(24.8)$ \\
\hline \multicolumn{2}{|c|}{ Age at diagnosis of mesothelioma, years } \\
\hline$\leq 39$ & $0(0)$ \\
\hline $40-49$ & $5(5.0)$ \\
\hline $50-59$ & $21(20.8)$ \\
\hline $60-69$ & $45(44.6)$ \\
\hline $70-79$ & $24(23.8)$ \\
\hline$\geq 80$ & $6(5.9)$ \\
\hline \multicolumn{2}{|l|}{ Mesothelioma site } \\
\hline Pleural & $93(92.1)$ \\
\hline Peritoneal & $8(7.9)$ \\
\hline \multicolumn{2}{|l|}{ Histology } \\
\hline Epithelioid & 79 (78.2) \\
\hline Biphasic & $7(6.9)$ \\
\hline Sarcomatoid & $10(9.9)$ \\
\hline Unknown & $5(5.0)$ \\
\hline \multicolumn{2}{|l|}{ Asbestos exposure $^{\dagger}$} \\
\hline Yes & $33(32.7)$ \\
\hline No & $68(67.3)$ \\
\hline
\end{tabular}

${ }^{\dagger}$, yes $=$ patients reported asbestos exposure; no $=$ patients ruled out or stated that they were not aware of asbestos exposure.

of DNA damage is caused by germline mutations in genes involved in DNA repair and HR, in the presence of the exposure to asbestos and other carcinogenic fibers, may be a driving force towards chromothripsis, as recently postulated by studying the germline risk alleles in multiple myeloma (47).

On the other hand, by using genome-wide long matepair sequencing in a family trio, intra-chromosomal rearrangements with the same pattern of chromothripsis were found as responsible for de novo structural alterations in germline DNA, suggesting that chromothripsis or a similar mechanism may be involved in chromosome shattering and non-homologous end joining (NHEJ), leading to genetic alterations involved in congenital defects (48) and possibly risk of developing cancer.

Therefore, genome-wide studies for the analysis of inherited genetic risk alterations in mesothelioma, where chromothripsis seems to play a relevant role, should include genes not only involved in DNA repair and HR pathways but also those involved in the mechanisms committed to maintaining the chromosomal integrity.

\section{Germline mutations in a Japanese cohort of patients with mesothelioma}

The association between occupational and even nonoccupational exposure to asbestos with mesothelioma has been established. Although the accurate determination of asbestos exposure is a difficult and complicated process, it is apparent from various epidemiological studies that only a relatively small fraction of subjects who had a history of asbestos exposure develop mesothelioma (4). Accumulating evidence in the field support the hypothesis that asbestos exposure triggers mesothelioma on the genetic background of moderate-risk at some tumor-related genes, which alone could not cause tumor, but raise the risk of developing mesothelioma.

To assess this hypothesis in different ethnicity, the germline genomic DNA of 101 Japanese patients was analyzed, including individuals with occupational and residential asbestos exposure, who were otherwise enrolled regardless of asbestos exposure, age at diagnosis, or personal or family history of cancer. Clinical data for this population is summarized in Table 2. tNGS was performed on 22 genes: BAP1, CDKN2A, CDKN2B, NF2, TP53, MLH1, CUL1, SETD2, SETBP1, and mammalian SWI/SNF (mSWI/ SNF) subunit genes, including PBRM1, ARID1A, ARID1B, ARID2, SMARCA2, SMARCA4, SMARCB1, SMARCC1, SMARCC2, SMARCD1, SMARCD2, SMARCD3, and SMARCE1.

The Human Genetic Variation Database (HGVD) provides a source of Japanese genetic variation based on the exome sequencing of 1,208 individuals and on genotyping data of common variations obtained from a cohort of 3,248 individuals (HGVD, http://www.hgvd.genome.med. kyoto-u.ac.jp). Also the Genome Aggregation Database (gnomAD, containing 125,748 exome sequences and 15,708 whole-genome sequences from unrelated individuals. previously known as the ExAC) was used to select germline variants that are absent or extremely rare and having a damaging protein score (>20 of CADD score) (Table 3). The results of our overall analysis revealed that 31 out of 101 subjects were carrying rare variants in 14 genes, total 25 
Table 3 Summary of the germline alterations found in the 101 patients with mesothelioma under study

\begin{tabular}{|c|c|c|c|c|c|c|}
\hline Gene & Variant & Chr & Pos (hg19) & $\begin{array}{l}\text { gnomAD } \\
\text { frequency }\end{array}$ & $\begin{array}{l}\text { HVGD } \\
\text { frequency }\end{array}$ & $\begin{array}{l}\text { No. patients } \\
\text { w. variants }\end{array}$ \\
\hline$B A P 1$ & G/A > Q436Ter & 3 & 52437855 & No hit & No hit & 1 \\
\hline$B A P 1$ & $\mathrm{G} / \mathrm{C}>\mathrm{S} 172 \mathrm{R}$ & 3 & 52441254 & No hit & No hit & 1 \\
\hline CDKN2A & $\mathrm{C} / \mathrm{T}>$ none $^{\dagger}$ & 9 & 21974676 & No hit & No hit & 1 \\
\hline$M L H 1$ & $\mathrm{G} / \mathrm{C}>\mathrm{V} 16 \mathrm{~L}$ & 3 & 37035084 & $1 / 251,438$ & No Hit & 1 \\
\hline$M L H 1$ & $\mathrm{~T} / \mathrm{G}>\mathrm{none}^{\dagger}$ & 3 & 37038202 & No hit & No hit & 1 \\
\hline MLH1 & $C / G>L 582 V / L 341 V$ & 3 & 37089022 & No hit & No hit & 1 \\
\hline SETBP1 & $\mathrm{C} / \mathrm{T}>\mathrm{R} 463 \mathrm{C}$ & 18 & 42530692 & $22 / 282,524$ & No Hit & 1 \\
\hline SETBP1 & $\mathrm{C} / \mathrm{T}>\mathrm{R} 1321 \mathrm{C}$ & 18 & 42533266 & $8 / 280,368$ & No hit & 1 \\
\hline$A R I D 1 B$ & $\mathrm{~A} / \mathrm{T}>\mathrm{M} 263 \mathrm{~L}$ & 6 & 157099850 & $1 / 119,342$ & No hit & 1 \\
\hline$A R I D 1 B$ & $\mathrm{C} / \mathrm{T}>\mathrm{P} 724 \mathrm{~S}$ & 6 & 157405967 & $33 / 282,730$ & 0.0008 & $1^{t+}$ \\
\hline ARID2 & $\mathrm{C} / \mathrm{T}>\mathrm{P} 1073 \mathrm{~S}$ & 12 & 46245123 & No hit & No hit & 1 \\
\hline ARID2 & $T / G>C 1271 G$ & 12 & 46245717 & No hit & No hit & 1 \\
\hline ARID2 & $\mathrm{A} / \mathrm{C}>\mathrm{K} 1627 \mathrm{~N}$ & 12 & 46254691 & $6 / 242,508$ & No hit & 2 \\
\hline SMARCD2 & $\mathrm{C} / \mathrm{G}>\mathrm{R} 357 \mathrm{P}$ & 17 & 61911540 & $3 / 237,020$ & No hit & 1 \\
\hline SMARCD3 & $\mathrm{C} / \mathrm{T}>\mathrm{R} 236 \mathrm{H}$ & 7 & 150938994 & $7 / 246,578$ & No hit & 1 \\
\hline
\end{tabular}

${ }^{\dagger}$, splice-donor site variant; ${ }^{\dagger \dagger}$, homozygous variant.

variants, and 3 subjects had 2 variants in each different gene combination. And $14 / 25$ of them were reported neither in the HGVD nor in the gnomAD.

In the $B A P 1$ gene, two loss-of-function mutations were found in two patients, and one possible pathogenic missense mutation was detected in an additional patient. This latter missense agC/agG substitution causes S172R amino acid change that has been classified as a pathogenic variant in the COSMIC (Catalogue Of Somatic Mutations In Cancer) database (https://cancer.sanger.ac.uk/cosmic), by using the Functional Analysis through Hidden Markov Models (FATHMM) prediction tool.

Other splice-donor site mutations were detected in $C D K N 2 A$ and $M L H 1$. Surprisingly, 12 rare missense variants were found in the mSWI/SNF subunit genes in patients with mesothelioma. The mSWI/SNF chromatin remodeling complex, required for the transcription of genes repressed by chromatin, is made of 12 - to 15 -subunit macromolecular assemblies, which are the products of 29 different genes. These genes are involved in the differentiation of many 
lineages and in modulating transcriptional activity $(49,50)$. Frequent somatic mutations of these genes were found across a wide range of human cancers $(49,50)$, including mesothelioma (34). The occurrence of frequent germline missense variants in the $\mathrm{mSWI} / \mathrm{SNF}$ subunit genes family in our Japanese patients cohort indicates a possible role of these genes in the predisposition for mesothelioma.

Germline variants under the above criteria of frequency and CADD score $>20$ were also found in the lysine-specific histone methyltransferase genes, SETD2 and SETDB1. Inactivating somatic mutations of these epigenetic modifier genes have been previously found as prevalent in several cancers, including clear cell renal cell carcinoma and mesothelioma $(51,52)$. Moreover, several germline homozygous variants in the $\mathrm{mSWI} / \mathrm{SNF}$ subunit gene and SETD2 have been detected in mesothelioma (34). The germline variants identified in the Japanese patients with mesothelioma might not represent a major factor in pathogenesis but could be a modifying factor in the predisposition for mesothelioma.

\section{Conclusions}

The discovery of $B A P 1$ as a predisposition gene for the development of familial mesothelioma and other cancers (29) highly increased the number of genomic studies conducted on germline samples from patients with mesothelioma and individuals at risk. The most recent results generated novel important knowledge on the prevalence of mesotheliomas attributable to inherited mutations of susceptibility genes. In all studies BAP1 germline mutations were most prevalent, but also other tumor-linked genes were identified as frequently affected with germline alterations associated to the predisposition to mesothelioma, like those involved in DNA repair and HR $(20,38,53)$. A tNGS study identified a subset of patients with familial and early-onset mesothelioma characterized by improved survival, carrying germline mutations both in BAP1 and in HR DNA repair or tumor suppressor genes, further expanding the spectrum of the possible predisposition genes (33). Similar results were obtained in different studies (41), with the interesting finding of frequent pathogenic variants within the gene family associated with Fanconi's anemia cascade (42). The novel results presented here, obtained in a cohort of Japanese patients with mesothelioma, also show deleterious variants occurring in genes involved in the DNA repair and, for the first time, the occurrence of rare missense variants in the genes of the subunits assembling the mSWI/SNF chromatin remodeling complex.

Further studies will be required to portray a more precise picture of the genetic background required for the development of mesothelioma in subjects with a very high frequency of either pathogenic variants or moderate-risk germline variants, and to identify the full body of the genes predisposing to mesothelioma.

\section{Acknowledgments}

Funding: This work was supported in part by a Grant-inAid for Scientific Research (KAKENHI, 26460689 and 18K07212), a Grant-in-Aid for Researchers from Hyogo College of Medicine, 2017, to Y Yoshikawa.

\section{Footnote}

Conflicts of Interest: The authors have no conflicts of interest to declare.

Ethical Statement: The authors are accountable for all aspects of the work in ensuring that questions related to the accuracy or integrity of any part of the work are appropriately investigated and resolved.

Open Access Statement: This is an Open Access article distributed in accordance with the Creative Commons Attribution-NonCommercial-NoDerivs 4.0 International License (CC BY-NC-ND 4.0), which permits the noncommercial replication and distribution of the article with the strict proviso that no changes or edits are made and the original work is properly cited (including links to both the formal publication through the relevant DOI and the license). See: https://creativecommons.org/licenses/by-nc$\mathrm{nd} / 4.0 /$.

\section{References}

1. Sluis-Cremer GK, Liddell FD, Logan WP, et al. The mortality of amphibole miners in South Africa, 1946-80. Br J Ind Med 1992;49:566-75.

2. Franke K, Paustenbach D. Government and Navy knowledge regarding health hazards of asbestos: a state of the science evaluation (1900 to 1970). Inhal Toxicol 2011;23 Suppl 3:1-20.

3. Magnani C, Dalmasso P, Biggeri A, et al. Increased risk of malignant mesothelioma of the pleura after residential 
or domestic exposure to asbestos: a case-control study in Casale Monferrato, Italy. Environ Health Perspect 2001;109:915-9.

4. Carbone M, Adusumilli PS, Alexander HRJ, et al. Mesothelioma: Scientific Clues for Prevention, Diagnosis, and Therapy. CA Cancer J Clin 2019;69:402-29.

5. Baumann F, Ambrosi JP, Carbone M. Asbestos is not just asbestos: an unrecognised health hazard. Lancet Oncol 2013;14:576-8.

6. Carbone M, Baris YI, Bertino P, et al. Erionite exposure in North Dakota and Turkish villages with mesothelioma. Proc Natl Acad Sci U S A 2011;108:13618-23.

7. Yang H, Rivera Z, Jube S, et al. Programmed necrosis induced by asbestos in human mesothelial cells causes high-mobility group box 1 protein release and resultant inflammation. Proc Natl Acad Sci U S A 2010;107:12611-6.

8. Qi F, Okimoto G, Jube S, et al. Continuous exposure to chrysotile asbestos can cause transformation of human mesothelial cells via HMGB1 and TNF-alpha signaling. Am J Pathol 2013;183:1654-66.

9. Kadariya Y, Menges CW, Talarchek J, et al. InflammationRelated IL1 $\beta /$ IL1R Signaling Promotes the Development of Asbestos-Induced Malignant Mesothelioma. Cancer Prev Res (Phila) 2016;9:406-14.

10. Carbone M, Yang H. Mesothelioma: recent highlights. Ann Transl Med 2017;5:238.

11. Carbone M, Emri S, Dogan AU, et al. A mesothelioma epidemic in Cappadocia: scientific developments and unexpected social outcomes. Nat Rev Cancer 2007;7:147-54.

12. Roushdy-Hammady I, Siegel J, Emri S, et al. Geneticsusceptibility factor and malignant mesothelioma in the Cappadocian region of Turkey. Lancet 2001;357:444-5.

13. Testa JR, Cheung M, Pei J, et al. Germline BAP1 mutations predispose to malignant mesothelioma. Nat Genet 2011;43:1022-5.

14. Wiesner T, Obenauf AC, Murali R, et al. Germline mutations in BAP1 predispose to melanocytic tumors. Nat Genet 2011;43:1018-21.

15. Napolitano A, Pellegrini L, Dey A, et al. Minimal asbestos exposure in germline BAP1 heterozygous mice is associated with deregulated inflammatory response and increased risk of mesothelioma. Oncogene 2016;35:1996-2002.

16. Abdel-Rahman MH, Pilarski R, Cebulla CM, et al. Germline BAP1 mutation predisposes to uveal melanoma, lung adenocarcinoma, meningioma, and other cancers. J
Med Genet 2011;48:856-9.

17. Carbone M, Ferris LK, Baumann F, et al. BAP1 cancer syndrome: malignant mesothelioma, uveal and cutaneous melanoma, and MBAITs. J Transl Med 2012;10:179.

18. Murali R, Wilmott JS, Jakrot V, et al. BAP1 expression in cutaneous melanoma: a pilot study. Pathology 2013;45:606-9.

19. Kittaneh M, Berkelhammer C. Detecting germline BAP1 mutations in patients with peritoneal mesothelioma: benefits to patient and family members. J Transl Med 2018;16:194.

20. Panou V, Gadiraju M, Wolin A, et al. Frequency of Germline Mutations in Cancer Susceptibility Genes in Malignant Mesothelioma. J Clin Oncol 2018;36:2863-71.

21. Rai K, Pilarski R, Cebulla CM, et al. Comprehensive review of BAP1 tumor predisposition syndrome with report of two new cases. Clin Genet 2016;89:285-94.

22. Peña-Llopis S, Vega-Rubin-de-Celis S, Liao A, et al. BAP1 loss defines a new class of renal cell carcinoma. Nat Genet 2012;44:751-9.

23. Popova T, Hebert L, Jacquemin V, et al. Germline BAP1 mutations predispose to renal cell carcinomas. Am J Hum Genet 2013;92:974-80.

24. Njauw CN, Kim I, Piris A, et al. Germline BAP1 inactivation is preferentially associated with metastatic ocular melanoma and cutaneous-ocular melanoma families. PLoS One 2012;7:e35295.

25. de la Fouchardière $\mathrm{A}$, Cabaret $\mathrm{O}$, Savin L, et al. Germline BAP1 mutations predispose also to multiple basal cell carcinomas. Clin Genet 2015;88:273-7.

26. Wadt KA, Aoude LG, Johansson P, et al. A recurrent germline BAP1 mutation and extension of the BAP1 tumor predisposition spectrum to include basal cell carcinoma. Clin Genet 2015;88:267-72.

27. Shankar GM, Abedalthagafi M, Vaubel RA, et al. Germline and somatic BAP1 mutations in high-grade rhabdoid meningiomas. Neuro Oncol 2017;19:535-45.

28. Pilarski R, Cebulla CM, Massengill JB, et al. Expanding the clinical phenotype of hereditary BAP1 cancer predisposition syndrome, reporting three new cases. Genes Chromosomes Cancer 2014;53:177-82.

29. Carbone M, Yang H, Pass HI, et al. BAP1 and cancer. Nat Rev Cancer 2013;13:153-9.

30. Guénard F, Labrie Y, Ouellette G, et al. Genetic sequence variations of BRCA1-interacting genes AURKA, BAP1, BARD1 and DHX9 in French Canadian families with high risk of breast cancer. J Hum Genet 2009;54:152-61.

31. Pilarski R, Rai K, Cebulla C, et al. BAP1 Tumor 
Predisposition Syndrome. In: Adam MP, Ardinger HH, Pagon RA, et al. editors. GeneReviews ${ }^{\circledR}$. Seattle (WA): University of Washington, Seattle. 2016.

32. Walpole S, Pritchard AL, Cebulla CM, et al. Comprehensive Study of the Clinical Phenotype of Germline BAP1 Variant-Carrying Families Worldwide. J Natl Cancer Inst 2018;110:1328-41.

33. Pastorino S, Yoshikawa Y, Pass HI, et al. A Subset of Mesotheliomas With Improved Survival Occurring in Carriers of BAP1 and Other Germline Mutations. J Clin Oncol 2018. [Epub ahead of print].

34. Yoshikawa Y, Sato A, Tsujimura T, et al. Biallelic germline and somatic mutations in malignant mesothelioma: multiple mutations in transcription regulators including mSWI/SNF genes. Int J Cancer 2015;136:560-71.

35. Carbone M, Klein G, Gruber J, et al. Modern criteria to establish human cancer etiology. Cancer Res 2004;64:5518-24.

36. Vogel WH. Li-Fraumeni Syndrome. J Adv Pract Oncol 2017;8:742-6.

37. Cunniff C, Bassetti JA, Ellis NA. Bloom's Syndrome: Clinical Spectrum, Molecular Pathogenesis, and Cancer Predisposition. Mol Syndromol 2017;8:4-23.

38. Betti M, Aspesi A, Ferrante D, et al. Sensitivity to asbestos is increased in patients with mesothelioma and pathogenic germline variants in BAP1 or other DNA repair genes. Genes Chromosomes Cancer 2018;57:573-83.

39. Betti M, Casalone E, Ferrante D, et al. Germline mutations in DNA repair genes predispose asbestosexposed patients to malignant pleural mesothelioma. Cancer Lett 2017;405:38-45.

40. Richards S, Aziz N, Bale S, et al. Standards and guidelines for the interpretation of sequence variants: a joint consensus recommendation of the American College of Medical Genetics and Genomics and the Association for Molecular Pathology. Genet Med 2015;17:405-24.

41. Hassan R, Morrow B, Thomas A, et al. Inherited predisposition to malignant mesothelioma and overall survival following platinum chemotherapy. Proc Natl Acad Sci U S A 2019;116:9008-13.

42. Bertelsen B, Tuxen IV, Yde CW, et al. High frequency of pathogenic germline variants within homologous recombination repair in patients with advanced cancer. NPJ Genom Med 2019;4:13.

43. Ly P, Cleveland DW. Rebuilding Chromosomes After Catastrophe: Emerging Mechanisms of Chromothripsis. Trends Cell Biol 2017;27:917-30.

44. Yoshikawa Y, Emi M, Hashimoto-Tamaoki T, et al. Highdensity array-CGH with targeted NGS unmask multiple noncontiguous minute deletions on chromosome 3 p21 in mesothelioma. Proc Natl Acad Sci U S A 2016;113:13432-7.

45. Oey H, Daniels M, Relan V, et al. Whole-genome sequencing of human malignant mesothelioma tumours and cell lines. Carcinogenesis 2019;40:724-34.

46. Mansfield AS, Peikert T, Smadbeck JB, et al. Neoantigenic Potential of Complex Chromosomal Rearrangements in Mesothelioma. J Thorac Oncol 2019;14:276-87.

47. Janz S, Zhan F, Sun F, et al. Germline Risk Contribution to Genomic Instability in Multiple Myeloma. Front Genet 2019;10:424.

48. Kloosterman WP, Guryev V, van Roosmalen M, et al. Chromothripsis as a mechanism driving complex de novo structural rearrangements in the germline. Human Molecular Genetics 2011;20:1916-24.

49. Pulice JL, Kadoch C. Composition and Function of Mammalian SWI/SNF Chromatin Remodeling Complexes in Human Disease. Cold Spring Harb Symp Quant Biol 2016;81:53-60.

50. Wilson BG, Roberts CW. SWI/SNF nucleosome remodellers and cancer. Nat Rev Cancer 2011;11:481-92.

51. Kang HC, Kim HK, Lee S, et al. Whole exome and targeted deep sequencing identify genome-wide allelic loss and frequent SETDB1 mutations in malignant pleural mesotheliomas. Oncotarget 2016;7:8321-31.

52. Li J, Duns G, Westers H, et al. SETD2: an epigenetic modifier with tumor suppressor functionality. Oncotarget 2016;7:50719-34.

53. Betti M, Aspesi A, Biasi A, et al. CDKN2A and BAP1 germline mutations predispose to melanoma and mesothelioma. Cancer Lett 2016;378:120-30.
Cite this article as: Yoshikawa Y, Emi M, Nakano T, Gaudino G. Mesothelioma developing in carriers of inherited genetic mutations. Transl Lung Cancer Res 2020;9(Suppl 1):S67-S76. doi: 10.21037/tlcr.2019.11.15 